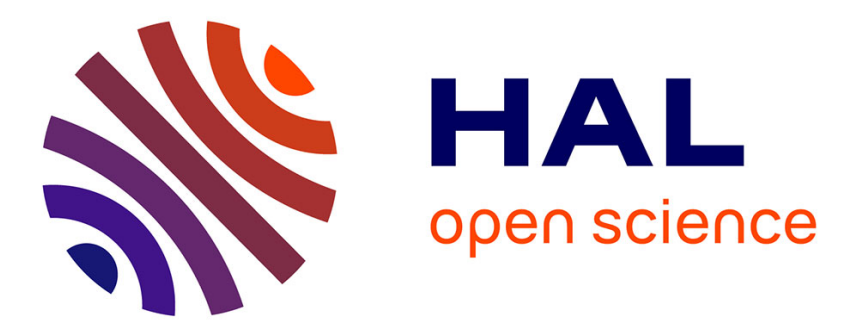

\title{
A supervisory control policy over an acoustic communication network
}

Alireza Farhadi, Jonathan Dumon, Carlos Canudas de Wit

\section{To cite this version:}

Alireza Farhadi, Jonathan Dumon, Carlos Canudas de Wit. A supervisory control policy over an acoustic communication network. International Journal of Control, 2015, 88 (5), pp.946-958. 10.1080/00207179.2014.986201 . hal-01132426

\section{HAL Id: hal-01132426 https://hal.science/hal-01132426}

Submitted on 17 Mar 2015

HAL is a multi-disciplinary open access archive for the deposit and dissemination of scientific research documents, whether they are published or not. The documents may come from teaching and research institutions in France or abroad, or from public or private research centers.
L'archive ouverte pluridisciplinaire HAL, est destinée au dépôt et à la diffusion de documents scientifiques de niveau recherche, publiés ou non, émanant des établissements d'enseignement et de recherche français ou étrangers, des laboratoires publics ou privés. 


\title{
A Supervisory Control Policy Over an Acoustic Communication Network
}

\author{
Alireza Farhadi, Jonathan Dumon and Carlos Canudas-de-Wit
}

\begin{abstract}
This paper presents a supervisory multi - agent control policy over an acoustic communication network subject to imperfections (packet dropout and transmission delay) for localization of an underwater flow source (e.g., source of chemical pollution, fresh water, etc.) with an unknown location at the bottom of the ocean. A two-loop control policy combined with a coding strategy for reliable communication is presented to perform the above task. A simulator is developed and used to evaluate the trade-offs between quality of communication, transmission delay and control for a fleet of Autonomous Underwater Vehicles (AUVs) supervised over a noisy acoustic communication network by an Autonomous Surface Vessel (ASV). It is illustrated that without compensation of the effects of severe random packet dropout, localization of an unknown underwater flow source is not possible for the condition simulated just by implementing a two-loop control policy. But, a two-loop control policy combined with a strategy for reliable communication locates the unknown location of flow source.
\end{abstract}

\section{Index Terms}

Networked control system, multi-agent system, supervisory control.

A. Farhadi is an Assistant Professor in the Department of Electrical Engineering, Sharif University of Technology, Tehran, Iran, email: afarhadi@sharif.edu

J. Dumon is a Research Engineer at GIPSA-Lab., Grenoble, France, email: jonathan.dumon@ gipsa-lab.grenoble-inp.fr

C. Canudas-de-Wit is the NeCS team CNRS director of research at CNRS - GIPSA-Lab./INRIA, Grenoble, France, email: carlos.canudas-de-wit@gipsa-lab.fr

The authors thank their industrial partner Ifremer (French institute for ocean research) for information on its AsterX autonomous underwater vehicles. 


\section{INTRODUCTION}

\section{A. Motivation and Background}

There are advantages in using a fleet of simple autonomous agents instead of one sophisticated complex agent, in terms of quality of the gathered information, robustness against unexpected events, scalability, etc. The fleet system is scalable, the number of agents forming the fleet can be adapted to a given task and its constraints, and the redundancy of a multi-agent system increases the operational reliability. Moreover, distributed sampling for spatial analysis and complementary information gathered by different sensor packages on different agents results in the realization of tasks by a fleet system that cannot be easily achieved by single agent systems.

In addition, in some applications, such as the extremum source seeking via the non-modelbased gradient estimation method, single agent systems cannot arbitrarily maneuver in all directions while simultaneously estimating the gradient. However, the center of the fleet system in a circular formation can arbitrarily maneuver in all direction while agents simultaneously estimate the gradient. This is a required assumption to solve the extremum source seeking problem via the non-model-based gradient estimation method.

Hence, in recent years, fleets of autonomous agents have been in the sharp attention of research as well as industry communities . Existing multi-agent algorithms for coordinating fleets of autonomous agents (e.g., [1] - [4]) are distributed algorithms. However, there is an increasing interest in supervisory multi-agent algorithms for coordinating fleets of autonomous agents. A supervisory multi-agent policy enables on board planning, monitoring, and intervention in case of error/emergency. Consequently, our national research center partner, Ifremer ${ }^{1}$, is interested in developing a fleet of Autonomous Underwater Vehicles (AUVs) supervised by an Autonomous Surface Vessel (ASV) to localize an underwater flow source (e.g., source of fresh water, chemical pollution, etc.) with unknown location (see Fig. 1). Our contributions to this problem so far are algorithms presented in [1], [2] that form a circular formation of mobile autonomous agents with a time varying center; and other algorithms presented in [3], [4] that move the center of formation in the direction of the gradient of concentration flow intensity. In particular, in [3] the authors presented a two-loop control policy that localizes the unknown underwater flow source when concentration flow intensity has elliptical level sets. Note that [3] is not concerned with

\footnotetext{
${ }^{1}$ French institute for ocean research. This institute performs underwater operations for ocean science purposes.
} 


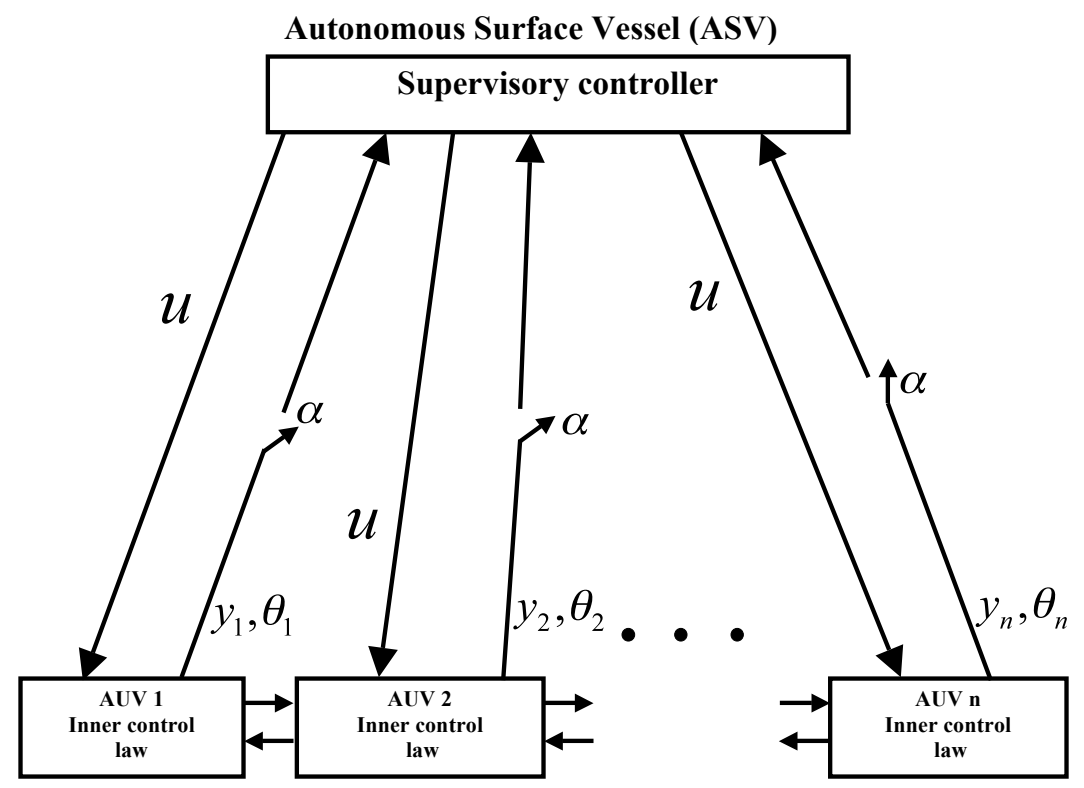

Fig. 1. Two-loop control strategy with inner and outer control loops for a fleet of AUVs supervised by an Autonomous Surface Vessel (ASV). Inner control loop: AUVs use a distributed control law that forms a uniformly distributed circular formation of AUVs with a time varying center. Outer control loop: AUVi, $i \in\{1,2, \ldots, n\}$, sends its sampled concentration data $y_{i}$ and rotational angle $\theta_{i}$ via a wireless link subject to transmission delay and random packet dropout with erasure probability $\alpha \neq 0$ to a remote ASV that produces accordingly the control input $\mathbf{u}$ to be used to update the position of the center of formation.

communication imperfections.

\section{B. Paper Contribution}

Underwater communication is usually wireless and via acoustic modems, in which this type of communication is generally subject to transmission noise. Using classical error detection techniques such as Checksum algorithm, the underwater acoustic channel can be modeled as a packet erasure channel with erasure probability $\alpha$. That is, with probability $\alpha$, a received packet of data (containing information about measurements, etc.) that has been corrupted by noise cannot be recovered and is erased.

For inter - AUV communication if the distance between underwater vehicles is small (which is the case in this paper), then transmission is subject to small attenuation resulting in a communication with large signal to noise ratio particularly as in deep water the noise mainly comes from AUV itself (which produces low level acoustic noise). But, as the distance between the fleet and the ASV is very often large; and in particular, as receiver noise level at the ASV is 
very often high due to waves, reflections on the surface and other boats noise, communication from AUVs to the ASV is subject to packet dropout with erasure probability $\alpha \in[0.1,0.5]$. Nevertheless, in communication from the ASV to AUVs, signal to noise ratio can be large as the ASV can broadcast with its maximum power and receiver noise level at AUVs is low. Hence, in the supervisory control architecture of Fig. 1, communication between AUVs and from the ASV to AUVs is assumed lossless, while communication from each AUV to the ASV can be subject to packet dropout with erasure probability $\alpha \neq 0$. Therefore, the supervisory control architecture of Fig. 1 is an example of the combined control-communication problem [5]-[13].

This paper presents an emerging example for the combined control-communication problem, which is described by the supervisory control architecture of Fig. 1. It extends the results of [3] to cases with more realistic representation for concentration flow intensity and illustrates that in the presence of severe random packet dropout, the two-loop control policy presented in [3] is not able to locate the unknown location of the underwater flow source without compensation of the effects of severe random packet dropout. However, this policy equipped with a coding strategy that provides a good quality of communication with a relatively low transmission delay locates the unknown location of the underwater flow source even in the presence of sever random packet dropout. The combined control-communication policy involves a tuning parameter that affects the quality of control, quality of communication and transmission delay. Hence, it can be tuned such that the interactions between control, quality of communication and transmission delay are balanced so that the best performance is achieved. A lower bound for tuning this parameter is presented and a simulator is developed and used to evaluate the trade-offs between quality of communication, transmission delay and control for a fleet of AUVs equipped with this combined control - communication policy. Using this simulator, the interactions between quality of communication, transmission delay and control are balanced for the condition simulated so that the best performance is achieved. It is illustrated that the performance of the best strategy in the presence of severe packet dropout is quite close to the performance of the ideal case of no dropout.

\section{Paper Organization}

The paper is organized as follows. In Section II, the problem formulation is presented. Then, Section III presents a supervisory multi-agent control policy over an acoustic communication network subject to imperfections. Subsequently, in Section IV, the interactions between quality 
of communication, transmission delay and control are balanced for the condition simulated to have the best performance for a fleet of Ifremer's AsterX AUVs. Section V summarizes the contributions of the paper.

\section{Problem Formulation}

Throughout the paper certain conventions are used: "”" means "by definition equals", $c(t)$ denotes the value of signal $c$ at continuous time $t$ and $c[k](=c(k T)$, where $T$ is the sampling period) is the value of signal $c$ at sampling instant $k \in \mathbf{N}_{+} \doteq\{0,1,2,3, \ldots\}$. $\operatorname{Re}(\cdot)$ denotes the real part and $\operatorname{Im}(\cdot)$ the imaginary part of a complex number. Vectors/matrices are denoted by bold letters, inner product is defined by $\left\langle\mathbf{z}_{1}, \mathbf{z}_{2}\right\rangle \doteq \operatorname{Re}\left\{\mathbf{z}_{1}^{*} \mathbf{z}_{2}\right\}$ where the superscript $*$ denotes the conjugate transpose, and the Euclidean norm is denoted by $\|\cdot\| \cdot\lfloor a\rfloor$ denotes the largest integer less than or equal to a scalar $a$, and for the simplicity of notation the dependency of signal $c(t)$ to time index $t$ is dropped whenever confusion does not occur. Transpose of a vector/matrix $\mathbf{A}$ is denoted by $\mathbf{A}^{\prime}$ and the time derivative of signal $c(t)$ is denoted by $\dot{c}(t)$.

\section{A. The Fleet of AUVs}

This paper is concerned with a supervisory multi-agent control policy consisting of a fleet of Ifremer's medium size AsterX AUVs with $n \in\{4,6,8,10\}$ vehicles and an ASV, as shown in Fig. 1 that are used for localization of an underwater flow source with unknown location at the bottom of the ocean. Each AUV has a torpedo shape with the following specifications:

\begin{tabular}{|c|c|c|c|c|c|}
\hline Weight (air) & Length & Power & Forward velocity & Range & Operating depth \\
\hline $200 \mathrm{~kg}$ & $2.5 \mathrm{~m}$ & Batteries/typical life time of 5 hour & maximum $2 \mathrm{~m} / \mathrm{s}$ & maximum $36 \mathrm{~km}$ & maximum $3 \mathrm{~km}$ \\
\hline
\end{tabular}

TABLE I

Specifications of IFremer's Medium Size AsterX AUVs.

Each AUV is equipped with sophisticated sensors. Therefore, in this study we ignore the effect of measurement noise. In the presence of high level measurement noise, appropriate filters can be used.

The fleet is supervised by an ASV. For communication between vehicles two different types of acoustic modems are used: 


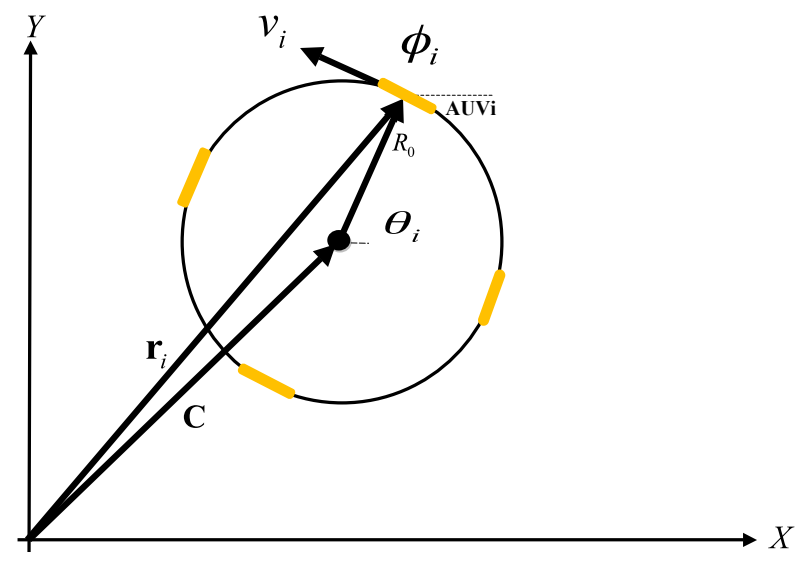

Fig. 2. A fleet of AUVs in a circular formation in the reference coordinate system. $\theta_{i}$ : rotational angle, $\phi_{i}$ : heading angle, $v_{i}$ : forward velocity, $\mathbf{c}$ : the center of formation vector, $\mathbf{r}_{i}$ : position vector, and $R_{0}$ : the radius of the circular formation.

- Type 1- Acoustic modems for communication between each AUV and the ASV are Sercel MATS 300 with the settings as given in Table II.

\begin{tabular}{|c|c|c|c|}
\hline Frequency & Bit rate & Transducer & Operating range \\
\hline $10 \mathrm{kHz}$ & $100 \mathrm{bits} / \mathrm{s}$ & Omni directional & maximum $3 \mathrm{~km}$ \\
\hline \multicolumn{4}{|c}{ TABLE II }
\end{tabular}

Modem Settings for COMmUnication betweEn Each AsterX AUV and the ASV.

- Type 2- Acoustic modems for inter - AUV communication are DSPComm with the settings as given in Table III.

\begin{tabular}{|c|c|c|c|}
\hline Frequency & Bit rate & Transducer & Bit error rate \\
\hline $10 \mathrm{kHz}$ & $480 \mathrm{bits} / \mathrm{s}$ & Omni directional & BER $=10^{-6}$ for the range of $150 \mathrm{~m}$ \\
\hline
\end{tabular}

MODEM SETTINGS FOR INTER-AUV COMMUNICATION.

Both type 1 and type 2 modems use Binary Phase Shift Keying (BPKS) modulation and maximum likelihood de-modulation schemes with ternary outputs: 0,1 , idle. 
The dynamics of each AUV is described by a 6 degrees of freedom model, which is subject to marine perturbations. However, the vehicles dynamics are handled by local control loops which are implemented within each vehicle. They result in a kinematic unicycle model for the fleet in a plane parallel to the ocean surface level, as follows:

$$
\dot{\mathbf{r}}_{i}=v_{i} \mathbf{e}\left(\phi_{i}\right), \dot{\phi}_{i}=u_{i}, \quad \mathbf{e}(\phi) \doteq\left(\begin{array}{c}
\cos \phi \\
\sin \phi
\end{array}\right), \quad i \in\{1,2, \ldots, n\},
$$

where $\mathbf{r}_{i}$ is the position vector, $\phi_{i}$ the heading angle, and the control inputs are the vehicle forward velocity $v_{i}>0$ and the turning rate $u_{i}$ (see Fig. 2$)^{2}$.

Throughout the paper, it is assumed that the source of concentration flow is located at the bottom of the ocean and the distribution of concentration flow intensity in the plane, where the fleet is located, is described by an unknown positive mapping $\rho$; and agent $i$ measures the local concentration flow intensity as $y_{i} \doteq \rho\left(\mathbf{r}_{i}\right)$.

\section{B. Formation Control (Inner Control Loop)}

In [1] an inner feedback control law that asymptotically stabilizes AUVs to a circular formation around a dynamic center point $\mathbf{c}(t)$ with uniformly distributed formation ${ }^{3}$ was proposed for $n(\geq 2)$ AUVs. With a desired forward velocity of $v_{0}$ and a desired rotational velocity of $\omega_{0}$, the feedback control law of [1] is given as follows:

$$
\begin{aligned}
& v_{i}=\left\|v_{0} \mathbf{e}\left(\psi_{i}\right)+\dot{\mathbf{c}}\right\|, \\
& u_{i}=\left(1-\frac{<\dot{\mathbf{r}}_{i}, \dot{\mathbf{c}}>}{<\dot{\mathbf{r}}_{i}, \dot{\mathbf{r}}_{i}>}\right)-\frac{\dot{\mathbf{r}}_{i}^{\prime} T(\pi / 2) \ddot{\mathbf{c}}}{<\dot{\mathbf{r}}_{i}, \dot{\mathbf{r}}_{i}>}, \\
& T(\phi)=\left(\begin{array}{cc}
\cos \phi & -\sin \phi \\
\sin \phi & \cos \phi
\end{array}\right), \\
& \dot{\psi}_{i}=w_{0}\left(1-\kappa<\mathbf{r}_{i}-\mathbf{c}, v_{0} \mathbf{e}\left(\psi_{i}\right)>\right)-\frac{\partial U}{\partial \psi_{i}}, i=1,2, \ldots, n,
\end{aligned}
$$

\footnotetext{
${ }^{2}$ For each AUV a local control loop implements a feedback linearization technique that replaces the vehicle nonlinear dynamics with a pseudo linear dynamics. Then, $H_{\infty}$ controllers are used to compensate the effects of model inaccuracy and perturbations, including marine perturbations, and force the vehicle parameters $\dot{r}_{i}$ and $\dot{\phi}_{i}$ to follow the desired set points $v_{i} \mathbf{e}\left(\phi_{i}\right)$ and $u_{i}$, respectively [14].

${ }^{3}$ That is, AUVs are uniformly distributed along a circumference by $\frac{2 \pi}{n}$ radians each.
} 


$$
U(\psi)=-\frac{K}{n} \sum_{m=1}^{\lfloor n / 2\rfloor} \frac{1}{2 m^{2}}<\left(\begin{array}{c}
\mathbf{e}\left(\psi_{1}\right) \\
\cdot \\
\cdot \\
\cdot \\
\mathbf{e}\left(\psi_{n}\right)
\end{array}\right), \overline{\mathbf{L}}\left(\begin{array}{c}
\mathbf{e}\left(\psi_{1}\right) \\
\cdot \\
\cdot \\
\cdot \\
\mathbf{e}\left(\psi_{n}\right)
\end{array}\right)>
$$

Here $\kappa>0, \psi_{i}(t)$ has the following initial condition: $\psi_{i}(0)=\tan ^{-1}\left(\frac{\operatorname{Im}\left(\dot{\mathbf{r}}_{i}(0)-\dot{\mathbf{c}}(0)\right)}{\operatorname{Re}\left(\dot{\mathbf{r}}_{i}(0)-\dot{\mathbf{c}}(0)\right)}+\epsilon_{i} \pi\right)$ where $\epsilon_{i}=0$ if $\operatorname{Re}\left(\dot{\mathbf{r}}_{i}(0)-\dot{\mathbf{c}}(0)\right) \geq 0$ and 1 otherwise, and $\overline{\mathbf{L}} \doteq \mathbf{L} \otimes \mathbf{I}_{2}$, where $\mathbf{L}$ is the Laplacian matrix associated with the communication network of AUVs, $\otimes$ denotes the Kronecker product and $\mathbf{I}_{2}$ is the $2 \times 2$ identity matrix.

Assuming that $\mathbf{c}(t)$ is twice differentiable, has bounded first and second time derivatives, and satisfies the following condition: $\sup _{t \geq 0}|\dot{\mathbf{c}}(t)|<v_{0}$, then, as shown in [1], the control inputs, as given above, steer AUVs to trajectories that lie on the circle with radius $R_{0}=v_{0} /\left|w_{0}\right|$ and time varying center $\mathbf{c}(t)$. Moreover, if $K>0$ and the communication network between AUVs is complete, then AUVs are uniformly distributed on that circle.

Remark 2.1: i) Throughout the paper it is assumed that the communication network between AUVs is complete. That is, every AUV talks to every other AUV; and hence, every AUV is connected to every other AUV.

ii) To form a uniformly distributed circular formation of AUVs with center c, parameters $\psi_{i}$, $i \in\{1,2, \ldots, n\}$, must be exchanged between AUVs.

iii) Parameters $\kappa$ and $K$ change the convergence rate to a circular formation and a uniform distribution of AUVs on the circular formation, respectively.

\section{Supervisory Multi-Agent Control Policy over Acoustic Communication Network (Outer Control Loop)}

In this section, by implementing the inner control loop (2) we are given a stable uniformly distributed circular formation of $n$ AUVs in a plane that is described by a center point $\mathbf{c}$, radius $R_{0}$, and an angle $\theta_{i}$ (see Fig. 2). In this circular formation, the position of each AUVi is described by the following equation: $\mathbf{r}_{i}=\mathbf{c}+R_{0} \mathbf{e}\left(\theta_{i}\right)$. The objective of this section is to provide an outer control loop policy located at the ASV equipped with a coding strategy for real-time reliable communication that moves the center of formation $\mathbf{c}$ towards the source of concentration flow in the plane where AUVs are located. This objective is fulfilled by updating $\dot{\mathbf{c}}$ in (2), as follows:

$$
\dot{\mathbf{c}}=\mathbf{u},
$$


where the control signal $\mathbf{u}$ is generated from the received rotational angles $\theta_{i}, i \in\{1,2, \ldots, n\}$, and sampled local concentration flow intensity $y_{i}, i \in\{1,2, \ldots, n\}$, taken by individual AUVs, which are transmitted to the ASV over an acoustic wireless communication network subject to packet dropout.

The outer control loop policy considered in this paper uses the output of a repetition coding strategy as its input. The repetition coding strategy can be easily implemented and for this reason it is used in this study. In the repetition coding strategy considered in this paper instead of sending one packet of data corresponding to each pair of measurements $\left(y_{i}, \theta_{i}\right), M$ identical packets of data are transmitted. Consequently, using this coding strategy the possibility of not recovering a pair of measurements at the ASV is reduced to $\alpha^{M}$.

In what follows the outer control loop policy is described and then the coding strategy in more detail.

\section{A. Outer Control Loop Design}

In this section, it is assumed that the outer control loop action is updated at the ASV, as follows:

$$
\mathbf{u}(t)=\left\{\begin{array}{lr}
\sum_{i=1}^{n} \rho\left(\mathbf{r}_{i}(t)\right) \mathbf{e}\left(\theta_{i}(t)\right) & \text { if all transmitted pairs to the ASV are recovered } \\
\sum_{i=1}^{n} \rho\left(\mathbf{r}_{i}\left(t^{-}\right)\right) \mathbf{e}\left(\theta_{i}\left(t^{-}\right)\right) & \text {Otherwise }
\end{array}\right.
$$

where $\theta\left(t^{-}\right)$denotes the previous update when all transmissions are successful. This is to say that when transmission to the ASV is not reliable, then $\mathbf{u}(t)$ is chosen to be equal to the previous outer control loop action that has been updated based on all successfully recovered transmitted pairs to the ASV.

In the following theorem borrowed from [3], it is shown that in the absence of communication imperfections when the concentration flow intensity has elliptical level sets, updating $\dot{\mathbf{c}}=\mathbf{u}$, as suggested by (4), moves the center of formation towards the location of the unknown flow source. In the following theorem it is assumed that for some positive definite matrix $\mathbf{A}, \rho$ is a continuously differential mapping and satisfies the following property:

$$
\begin{aligned}
\left(\mathbf{z}_{1}-\mathbf{c}^{*}\right)^{\prime} \mathbf{A}\left(\mathbf{z}_{1}-\mathbf{c}^{*}\right) & <\left(\mathbf{z}_{2}-\mathbf{c}^{*}\right)^{\prime} \mathbf{A}\left(\mathbf{z}_{2}-\mathbf{c}^{*}\right) \\
& \Rightarrow \\
\rho\left(\mathbf{z}_{1}\right) & >\rho\left(\mathbf{z}_{2}\right), \quad \mathbf{z}_{1}, \mathbf{z}_{2}, \mathbf{c}^{*} \in \mathbf{R}^{2} .
\end{aligned}
$$

This is to say that the signal strength has a unique maximum at a point $\mathbf{c}^{*}$ (the unknown fixed location of the source) and has compact elliptical level sets in the plane where the fleet is located. 
Theorem 3.1: [3] Suppose that even number of $n$ AUVs are given, the concentration flow intensity $\rho$ is a continuously differentiable mapping satisfying the property (5) and communication is free from imperfections. Suppose also that by implementing the inner control loop the $n$ AUVs form a stable uniformly distributed circular formation. Then, under the outer control loop action (4), the point $\mathbf{c}=\mathbf{c}^{*}$ is an asymptotically stable equilibrium of (3).

In reality the level sets of concentration flow intensity has more complex shape than elliptical shape considered in [3]. In fact, level sets that are resulted from the summation of a few elliptical sets (as shown in Fig. 5) are better representation for the level sets of concentration flow intensity. Therefore, in the following theorem we extend the results of [3] to the cases with more realistic representation for concentration flow intensity.

Theorem 3.2: Suppose that $n \in\{4,6,8,10, \ldots\}$ AUVs are given, communication is free from imperfections, by implementing the inner control loop the fleet system forms a stable uniformly distributed circular formation and the fleet system is under the action of the outer control loop policy (4). Then, the point $\mathbf{c}=\mathbf{c}^{*}$ is an asymptotically stable equilibrium of (3) for the concentration flow intensity of the following form

$$
\begin{array}{r}
\rho(\mathbf{z})=\beta_{1} e^{-\alpha_{1}\left(\mathbf{z}-\mathbf{c}^{*}\right)^{\prime} \mathbf{A}_{\mathbf{1}}\left(\mathbf{z}-\mathbf{c}^{*}\right)}+\ldots+\beta_{p} e^{-\alpha_{p}\left(\mathbf{z}-\mathbf{c}^{*}\right)^{\prime} \mathbf{A}_{\mathbf{p}}\left(\mathbf{z}-\mathbf{c}^{*}\right)}, \\
\alpha_{1}, \ldots, \alpha_{p}>0, \quad \beta_{1}, \ldots, \beta_{p} \geq 0, \quad \mathbf{A}_{1}, \ldots, \mathbf{A}_{\mathbf{p}}>0 .
\end{array}
$$

Proof: The proof follows from a Lyapunov stability argument involving the Lyapunov function $L(\mathbf{c})=\rho\left(\mathbf{c}^{*}\right)-\rho(\mathbf{c})$, numbering those AUVs that have a non-negative projection along the signal gradient from 1 to $\frac{n}{2}$ (i.e., for $\mathrm{AUVi}, i=1,2, \ldots, \frac{n}{2}, \nabla \rho^{\prime}(\mathbf{c}) \mathbf{e}\left(\theta_{i}\right) \geq 0$ ), the fact that as $n$ is even and AUVs are uniformly distributed (see Fig. 2), $\mathbf{r}_{i}, \mathbf{c}$ and $\mathbf{r}_{i+\frac{n}{2}}$ all lie along the same line, and the projection of the quadratic function $\left(\mathbf{z}-\mathbf{c}^{*}\right)^{\prime} \mathbf{A}_{\mathbf{j}}\left(\mathbf{z}-\mathbf{c}^{*}\right), j=1,2, \ldots, p$, evaluated along this line is a parabola with its minimum corresponding to a point in the direction of $\mathbf{r}_{i}$ from $\mathbf{c}$. The detail of the proof is as follows: For each $\mathbf{A}_{j}>0, j=1,2, \ldots, p$, the quadratic function $q_{j}(\mathbf{z}) \doteq\left(\mathbf{z}-\mathbf{c}^{*}\right)^{\prime} \mathbf{A}_{j}\left(\mathbf{z}-\mathbf{c}^{*}\right)$ evaluated along the line that connects $\mathbf{r}_{i}, \mathbf{c}$ and $\mathbf{r}_{i+\frac{n}{2}}$ is a parabola with its minimum corresponding to a point in the direction of $\mathbf{r}_{i}$ from $\mathbf{c}$. Due to the symmetry of this parabola and as $\mathbf{r}_{i}$ is closer to its minimum, it is the case that $\left(\mathbf{r}_{i}-\mathbf{c}^{*}\right)^{\prime} \mathbf{A}_{j}\left(\mathbf{r}_{i}-\mathbf{c}^{*}\right) \leq$ $\left(\mathbf{r}_{i+\frac{n}{2}}-\mathbf{c}^{*}\right)^{\prime} \mathbf{A}_{j}\left(\mathbf{r}_{i+\frac{n}{2}}-\mathbf{c}^{*}\right)$, in which from (6) it implies that $\rho\left(\mathbf{r}_{i}\right) \geq \rho\left(\mathbf{r}_{i+\frac{n}{2}}\right)$. Consequently, $\dot{L}(\mathbf{c})=-\sum_{i=1}^{\frac{n}{2}}\left(\rho\left(\mathbf{r}_{i}\right)-\rho\left(\mathbf{r}_{i+\frac{n}{2}}\right)\right) \nabla \rho^{\prime}(\mathbf{c}) \mathbf{e}\left(\theta_{i}\right) \leq 0$. The only situation in which $\dot{L}(\mathbf{c})=0$ for $\mathbf{c} \neq \mathbf{c}^{*}$ is the case when $n=2$ and the AUVs displacement vectors from the center of formation are orthogonal to $\nabla \rho(\mathbf{c})$. As we excluded the case of $n \neq 2$, then $\dot{L}(\mathbf{c})<0$ for $n \in\{4,6,8,10, \ldots\}$ AUVs considered in this theorem. Hence, under the outer control loop 
action (4), the point $\mathbf{c}=\mathbf{c}^{*}$ is an asymptotically stable equilibrium of (3) for the concentration flow intensity of the form (6).

\section{B. Coding Design}

As mentioned earlier, underwater transmission via acoustic modems is subject to random packet dropout in communication from AUVs to the ASV with erasure probability $\alpha$. If the underwater condition does not significantly change during search mission, the erasure probability $\alpha$ can be assumed fixed and it can be measured by transmitting test signals. Therefore, throughout it is assumed that the erasure probability is known a priori.

To compensate the effects of random packet dropout and improve the quality of communication from AUVs to the ASV, a repetition coding strategy is used. According to this strategy corresponding to each pair of measurements $\left(y_{i}, \theta_{i}\right)$, instead of sending just one packet of data, $M$ identical packets of data are sent. Therefore, using this strategy, the probability of not recovering a pair of measurements at the $\mathrm{ASV}$ is reduced to $\alpha^{M}$.

In addition of the random packet dropout underwater transmission via acoustic modems is also subject to the following major communication imperfections:

- long sound travel time $T_{d i}$ in communication between AUVi and the ASV, which is due to the slow propagation of underwater sound waves ${ }^{4}$. As this delay can be measured using test signals, throughout it is assumed that the sound travel time $T_{d i}$ is known a priori.

- limited communication bandwidth: The bit rate of the type 1 modems is $D_{1}=100 \mathrm{bits} / \mathrm{s}$ and the bit rate of the type 2 modems is $D_{2}=480$ bits/s. These limited communication bandwidths result in a long packets transmission time in communication of long packets of data 5 .

To avoid collision of transmitted information, a Time Division Multiple Access (TDMA) scheme [15] is used (see Fig. 3) ${ }^{6}$. The type 1 modems are used to transmit pairs $\left(y_{i}, \theta_{i}\right)$, $i \in\{1,2, \ldots, n\}$, from AUVs to the ASV, and control signal $\mathbf{u}$ from the ASV to AUVs. The type

\footnotetext{
${ }^{4}$ The speed of underwater sound waves is typically $v_{s}=1500 \mathrm{~m} / \mathrm{s}$; and therefore, if the distance between AUVi and the ASV is $d_{i}$, then $T_{d i}=d_{i} / v_{s}$ second.

${ }^{5}$ To exchange information (measurements, control commands, etc.) between vehicles with negligible distortion, long packets of data are used. Note that distortion is due to the quantization of information.

${ }^{6}$ Currently TDMA scheme is the only commercially available technique for underwater multiple accessing without collision.
} 


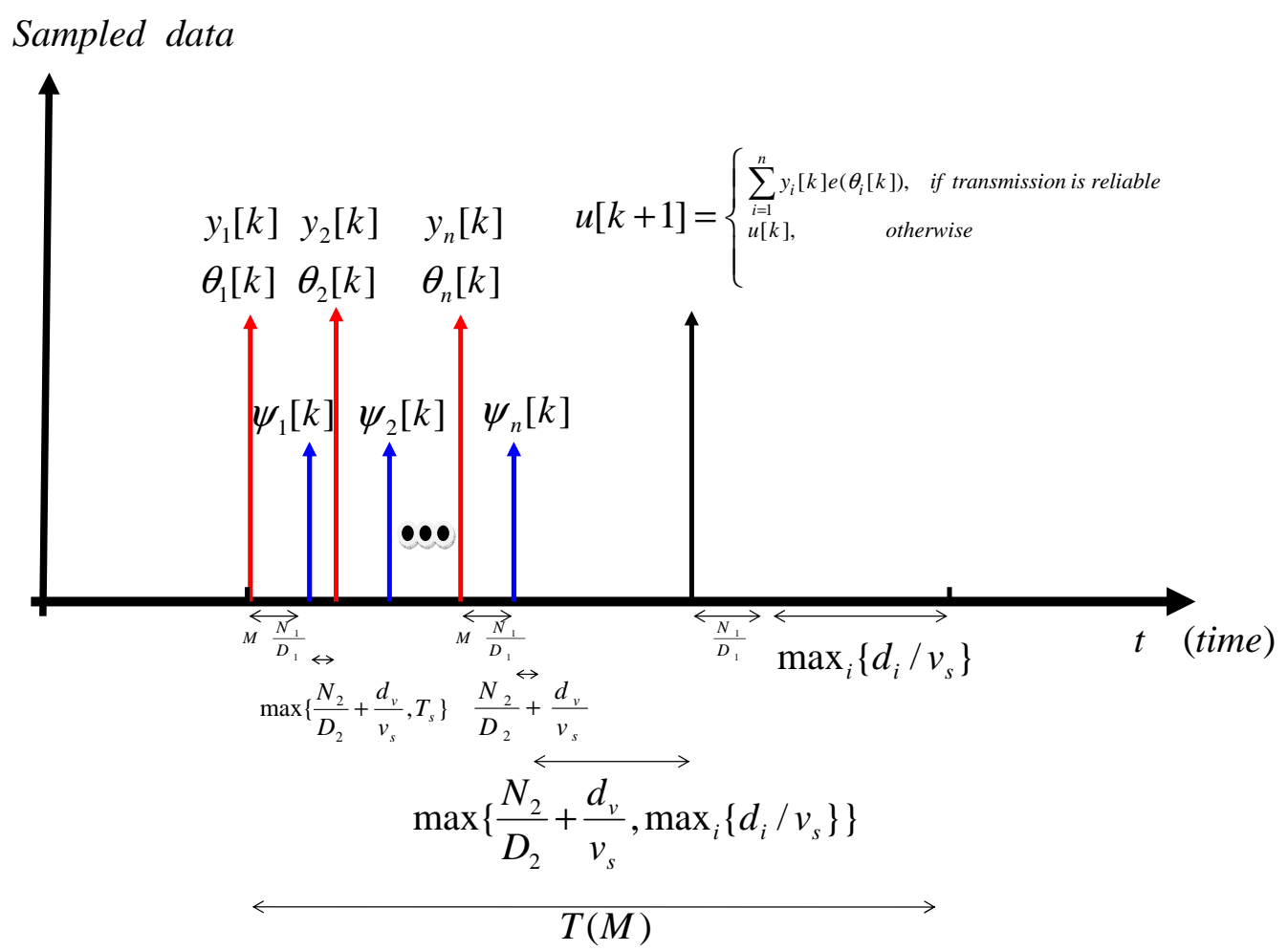

Fig. 3. A TDMA scheme used for exchanging information between the fleet of AUVs and the ASV without collision. $N_{1}$ : the length of packets of data representing pairs $\left(y_{i}, \theta_{i}\right)$ and $\mathbf{u}, N_{2}$ : the length of packets of data representing $\psi_{i}, M$ : the number of packet repetitions, $D_{1}$ : the bit rate of communication links between the fleet and the ASV, $D_{2}$ : the bit rate of inter-AUV communication links, $T_{s}$ : security time, $d_{v}$ : the maximum distance between AUVs, $d_{i}$ : the distance between AUVi and the ASV and $v_{s}$ : the speed of underwater sound waves.

2 modems are also used to exchange $\psi_{i}, i \in\{1,2, \ldots, n\}$, between AUVs.

As mentioned above to compensate the effects of packet dropout and have a reliable communication from each AUV to the ASV, a repetition coding strategy is used. That is, corresponding to each pair of measurements $\left(y_{i}, \theta_{i}\right)$, instead of sending just one packet of data with length $N_{1}, M$ identical packets of data are sent within an allocated time slot to each AUV, in which for each AUV the required time to transmit packets is $C(M) \doteq M \frac{N_{1}}{D_{1}}$ second, where $D_{1}$ is the communication bit rate. As it takes $C(M)$ second to transmit $M$ identical packets of data, $C(M)$ contributes to transmission time delay from AUVs to the ASV. Throughout, $C(M)$ is referred as packets transmission delay.

For a given fleet of AUVs, the trade-off between quality of communication that is presented in terms of the possibility of not recovering a pair of measurements at the ASV, i.e., $100 \alpha^{M}$ percent, and $M$ for a given $\alpha$, is geometric, while the trade-off between $M$ and $C(M)=M \frac{N_{1}}{D_{1}}$ 


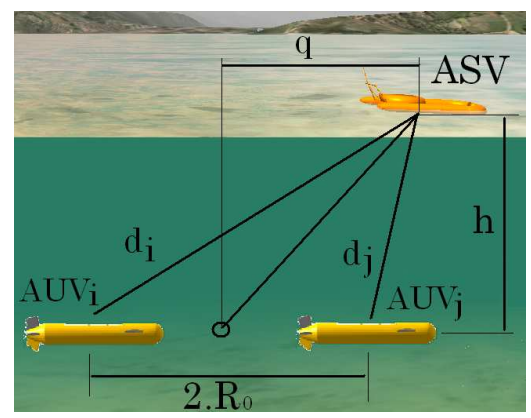

Fig. 4. A fleet of AUVs supervised by the ASV. $h$ : operating depth, $R_{0}$ : the radius of the circular formation, and $q$ : the distance between the ASV and the projection of the center of formation on the surface.

for $N_{1}=60$ bits and $D_{1}=100$ bits/s is linear. Hence, for a large $\alpha$ (e.g., $\alpha=0.5$ ) an excellent quality of communication demands a large transmission delay relative to the case of $M=1$.

As AUV and the ASV use embedded programming, the supervisory multi-agent control policy must be applied on discrete time domain. To avoid collision in transmission of information between vehicles, the sampling period $T(M)$ for updating outer control loop action is defined as follows (see Fig. 3):

$T(M)=n M \frac{N_{1}}{D_{1}}+(n-1) \max \left\{\frac{N_{2}}{D_{2}}+\frac{d_{v}}{v_{s}}, T_{s}\right\}+\max \left\{\frac{N_{2}}{D_{2}}+\frac{d_{v}}{v_{s}}, \max _{i}\left\{\frac{d_{i}}{v_{s}}\right\}\right\}+\frac{N_{1}}{D_{1}}+\max _{i} \frac{d_{i}}{v_{s}}$,

where $N_{2}$ is the length of packet corresponding to each $\psi_{i}\left(N_{2}=30 \mathrm{bits}\right), d_{v}$ is the maximum distance between AUVs ${ }^{7}, v_{s}=1500 m / s$ is the speed of underwater sound waves, $T_{s}$ is the security time to be defined shortly, and $d_{i}$ is the distance between AUVi and the ASV. Note that when the fleet operates at a high depth, then $d_{i} \approx h$, where $h$ is the operating depth of the fleet, provided the ASV follows the projection of the center of formation on the surface (see Fig. 4).

Remark 3.3: If the ASV moves exactly above the center of formation, transmission time delay from all AUVs to the ASV is the same. But, in reality this is not the case; and the ASV that follows the projection of the center of formation on the surface, can just be very close to it (see Fig. 4). In this case, as shown in Fig. 4, packets transmitted by AUVi are received by the ASV later than expected, while packets transmitted by AUVj are received earlier than expected. This

\footnotetext{
${ }^{7}$ When the formation is perfect, i.e., it is circular, $d_{v}=2 R_{0}$. But, as the formation may be disturbed during search mission due to perturbations, etc., $d_{v}$ is chosen to be $3 R_{0}$.
} 
may result in collision of data at the ASV. Therefore, to avoid this collision, the security time,

$$
T_{s}=\frac{\max _{i, j}\left(\left|d_{i}-d_{j}\right|\right)}{v_{s}}=\frac{\sqrt{\left(q+\frac{R_{0}}{2}\right)^{2}+h^{2}}-\sqrt{\left(q-\frac{R_{0}}{2}\right)^{2}+h^{2}}}{v_{s}},
$$

is added to $T(M)$ to compensate the time differences in propagation of packets from different AUVs.

If the sampling period $T(M)$ is sufficiently small, then a simple way to approximate the value of the center of formation in discrete time domain $\mathbf{c}[k]$ is as follows: $\mathbf{c}[k]=\mathbf{c}[k-1]+T(M) \mathbf{u}[k]$. Similarly, its first and second derivatives can be approximated, as follows: $\dot{\mathbf{c}}[k]=\mathbf{u}[k]$, and $\ddot{\mathbf{c}}[k]=\frac{\mathbf{u}[k]-\mathbf{u}[k-1]}{T(M)}$, respectively.

Remark 3.4: i) When a pair of measurements $\left(y_{i}, \theta_{i}\right)$ is not recovered at the ASV, the control input $\mathbf{u}[k]$ is updated with the last value: $\mathbf{u}[k]=\mathbf{u}[k-1]$.

ii) The angle of the outer control loop signal, i.e., $\angle \mathbf{u}[k], k \in \mathbf{N}_{+}$, represents the direction towards the unknown location of underwater flow source from the center point $\mathbf{c}[k]$. Therefore, to have the fastest convergence to the unknown source location, instead of using (3), AUVs can update $\dot{\mathbf{c}}[k]$ as follows: $\dot{\mathbf{c}}[k]=V_{\max } \angle \mathbf{u}[k]$, where $V_{\max }$ is the maximum possible speed of moving the center of formation with the possibility of maneuvering in all directions while AUVs simultaneously estimate the gradient. Subsequently, the next center of formation $\mathbf{c}[k]$ and $\ddot{\mathbf{c}}[k]$ are approximated by $\mathbf{c}[k]=\mathbf{c}[k-1]+T(M) V_{\max } \angle \mathbf{u}[k]$ and $\ddot{\mathbf{c}}[k]=\frac{V_{\max }(\angle \mathbf{u}[k]-\angle \mathbf{u}[k-1])}{T(M)}$, respectively. iii) In very long search missions, clock drift between AUVs must be estimated and an extra security time must be added to each AUV's cycle to avoid losing TDMA synchronization. As an example two quartz clocks with $\pm 30 \mathrm{ppm}$ precision lead to a drift of 1.08 second in every 5 hour (which is equal to the AUVs life time).

Definition 3.5: At each $k T(M)$ second, define a ball with center c and sufficiently large radius $r_{0}$. Then, it is said that the fleet system converges to $\mathbf{c}^{*}$ (or locates the unknown location of the source) if and only if the center of formation stays in this ball for enough time of $T_{0} \geq 10 T_{a}$ second after entering to this ball, where $T_{a}=\frac{r_{0}}{V_{\max }}$ second. In this case, $\mathbf{c}$ is an approximation for $\mathbf{c}^{*}$.

As each AUV has a limited life time, it is essential that the fleet locates the source of concentration flow as soon as possible. Therefore, the performance of the fleet is described by the time period between starting a mission and the time that the fleet finds the source. This time period is denoted by $T_{\text {total }}$. Obviously, a fleet with smaller $T_{\text {total }}$ has a better performance.

The following corollary extends the results of Theorem 3.2 by presenting a condition on the 
number of packet repetitions $M$ for localization of the source in the presence of communication packet dropout.

Corollary 3.6: Suppose that $n \in\{4,6,8,10\}$ AUVs are given, the concentration flow intensity $\rho$ is a continuously differentiable mapping described by (6) and communication from AUVs to the ASV is subject to packet dropout, as described earlier. Suppose also that by implementing the inner control loop the $n$ AUVs form a stable uniformly distributed circular formation and the repetition coding strategy as described above is used. Then, under the outer control loop action, the source is located if the number of packet repetitions $M$ satisfies the following condition:

$$
\left(1-\left(1-\alpha^{M}\right)^{n}\right)^{J(M)}<\frac{T_{a}}{T_{0}}, \quad J(M) \doteq\left\lfloor\frac{T_{a}}{T(M)}\right\rfloor, T_{a}=\frac{r_{0}}{V_{\max }} .
$$

Proof: When transmission to the ASV is not reliable (i.e., some of transmitted measurements are not recovered), from the description of the outer control loop policy (and in particular as the outer control loop action is not updated when packet dropout occurs and hence the center of formation continues moving along the previous direction) it follows that in most cases the time derivative of the Lyapunov function used in the proof of Theorem 3.2 at the center point $\mathbf{c}[k]$, i.e., $\dot{L}(\mathbf{c}[k]) \approx \frac{\Delta L(\mathbf{c}[k])}{T(M)}=\frac{L(\mathbf{c}[k])-L(\mathbf{c}[k-1])}{T(M)}$, will be still negative; and hence, the center of formation $\mathbf{c}$ continues moving towards $\mathbf{c}^{*}$. However, after entering the center of formation $\mathbf{c}$ into the ball with center $\mathbf{c}^{*}$ and radius $r_{0}$, if transmission to the ASV is not reliable while the center $\mathbf{c}$ reaches to $\mathbf{c}^{*}$, then the gradient signal $\nabla \rho(\mathbf{c}[k])$ will be in the opposite direction of $\dot{\mathbf{c}}[k]=\sum_{i=1}^{n} \rho\left(\mathbf{r}_{i}[k]\right) \mathbf{e}\left(\theta_{i}[k]\right)$; and therefore, $\dot{L}(\mathbf{c}[k])\left(\approx \frac{\Delta L(\mathbf{c}[k])}{T(M)}\right)=-\nabla \rho^{\prime}(\mathbf{c}[k]) \dot{\mathbf{c}}[k]$ will be positive. Consequently, $\mathbf{c}$ is getting away from $\mathbf{c}^{*}$ and exits the ball if during the time period $T_{a}=\frac{r_{0}}{V_{\max }}$ that the center $\mathbf{c}$ moves away from $\mathbf{c}^{*}$ with a constant speed of $V_{\max }$ all transmissions to the ASV are unsuccessful. Note that in this situation the function $L(\mathbf{c}[k])=\rho\left(\mathbf{c}^{*}\right)-\rho(\mathbf{c}[k])$ is an increasing function of time, in which from the description (6) for the concentration flow intensity $\rho$ it follows that the center of formation $\mathbf{c}$ is getting away from $\mathbf{c}^{*}$. To avoid such situations, the probability that all transmissions to the ASV are unsuccessful within the time period of $T_{a}$ second, i.e., $\left(1-\left(1-\alpha^{M}\right)^{n}\right)^{J(M)}$, where $J(M) \doteq\left\lfloor\frac{T_{a}}{T(M)}\right\rfloor$, must be small, e.g., less than $\frac{T_{a}}{T_{0}}$. This results in the condition (8) for $M$.

Extensive simulation study of the next section for different values of $\alpha$ illustrates that the above condition on the size of $M$ guarantees that the center of formation $\mathbf{c}$ enters to the ball with center $\mathbf{c}^{*}$ and stays there for enough time so that the source $\mathbf{c}^{*}$ is located. 
As discussed earlier, a larger $M$ results in a better quality of communication but as clear from Fig. 3 it also results in a larger packets transmission delay and therefore a longer time latency between making measurements and applying the control actions, which obviously damages the performance of both inner and outer control loops. Note that as the improvement in the quality of communication increases geometrically with $M$; while time latency increases linearly with $M$, a small increase in $M$ results in a significant improvement in the quality of communication; but a small increase in the time latency between making measurements and applying the control actions.

As shown in Corollary 3.6, in the presence of severe packet dropout, a small $M$ may not results in localization of the unknown underwater flow source. On the other hand, from Fig. 3 it follows that by increasing $M$, while the quality of communication improves significantly, the time latency between making measurements and applying the control actions on the fleet increases (linearly), which obviously damages the performance of the fleet system and results in a larger mission time $T_{\text {total }}$ such that for a very large $M$ the mission time may be larger than the AUVs life time. Therefore, in one extreme, small $M$ will not stabilize the fleet system particularly in the presence of severe random packet dropout, while for a bit larger $M$, the quality of communication is much better and hence localization of the underwater flow source with a relatively small time latency is achieved. But in other extreme, a very large $M$ results in a very long time latency and hence a large mission time that will be larger than AUVs life time, while for a smaller $M$, a search mission with smaller mission time is achieved. Hence, there exist an optimal $M^{*}$ between these two extremes that results in a good quality of communication with small time latency and therefore the smallest $T_{\text {total }}$. Simulation study of the next section for different values of $\alpha$ illustrates that for $\alpha=0.5, \alpha=0.3$ and $\alpha=0.1$, there exists a $M^{*}$.

\section{Simulation Results}

As shown in the previous sections, the supervisory multi-agent control policy of Fig. 1 is subject to two main communication imperfections: packet dropout in communication from AUVs to the ASV and transmission delay in communication between AUVs and the ASV. But, the effects of these drawbacks can be compensated by a proper adjustment of the number of packet repetitions $M$ so that the best performance in terms of the smallest mission time is achieved. This result is illustrated in the following section.

In this section for the purpose of illustration, the proposed supervisory multi-agent control 
policy over the acoustic communication network subject to packet dropout and transmission delay is applied to a fleet of Ifremer's medium size AsterX AUVs via MASim simulator developed in our research team in GIPSA-Lab. MASim is a MATLAB/SIMULINK simulator that simulates the behavior of fleets of Ifremer's AsterX AUVs in the actual environment. The fleet considered in the simulation study has the following specifications: $n=4, R_{0}=50 \mathrm{~m}, w_{0}=0.02 \mathrm{rad} / \mathrm{sec}$, $v_{0}=1 \mathrm{~m} / \mathrm{s}$ and $h=1500 \mathrm{~m}$. It is assumed that the concentration flow intensity at the horizontal plane parallel to the water surface level at the depth $h=1500 \mathrm{~m}$ (where the unknown source is located) is described as follows (note that AUVs are unaware of the concentration flow intensity description):

$$
\begin{gathered}
\rho(\mathbf{z})=\beta_{1} e^{-10^{-3}\left(\mathbf{z}-\mathbf{c}^{*}\right)^{\prime} \mathbf{A}_{1}\left(\mathbf{z}-\mathbf{c}^{*}\right)}+\beta_{2} e^{-10^{-3}\left(\mathbf{z}-\mathbf{c}^{*}\right)^{\prime} \mathbf{A}_{\mathbf{2}}\left(\mathbf{z}-\mathbf{c}^{*}\right)}+\beta_{3} e^{-10^{-3}\left(\mathbf{z}-\mathbf{c}^{*}\right)^{\prime} \mathbf{A}_{\mathbf{3}}\left(\mathbf{z}-\mathbf{c}^{*}\right)}, \\
\beta_{1}=5.6864, \quad \beta_{2}=17.0591, \quad \beta_{3}=57.2546, \\
\mathbf{A}_{1}=\left(\begin{array}{cc}
0.0014 & \frac{0.0045}{2} \\
\frac{0.0045}{2} & 0.855
\end{array}\right), \mathbf{A}_{2}=\left(\begin{array}{cc}
0.225 & -0.0045 \\
-0.0045 & 0.0135
\end{array}\right), \mathbf{A}_{3}=\left(\begin{array}{cc}
0.18 & \frac{-0.45}{2} \\
-\frac{0.45}{2} & 0.405
\end{array}\right) .
\end{gathered}
$$

Considering the transmission power level of type 1 and type 2 modems and the maximum distance between AUVs and the ASV, communication in the presence of low level noise (e.g., in deep water, where the noise mainly comes from AUV itself) can be considered almost lossless. Therefore, in the following simulations, just 5 percent packet loss is assumed to model communication between AUVs and from the ASV to AUVs. However, in the case of communication from AUVs to the ASV as the receiver is no longer in deep water and it is very close to the surface, it is subject to other sources of noise (e.g., waves, reflections on the surface and other boats noise) that dramatically degrades communication performance. Therefore, for communication from AUVs to the ASV much more packet loss, e.g., 50 percent packet loss (i.e., $\alpha=0.5$ ) is assumed. Moreover, it is assumed that there is a marine perturbation of 30 newton at the 50 degree angle with respect to the $\mathrm{X}$-axis of the reference coordinate system (see Fig. 2). Note that MASim simulator also include the effects of 6 degrees of freedom dynamics model of AUVs, local control loops and inner control loop in search mission.

To coordinate the fleet of AUVs towards the unknown location of the source in the presence of the above severe packet dropout in transmission from the fleet to the ASV, the supervisory multi-agent control policy of Section III is used. Table IV summarizes the parameters used for 
simulations $^{8}$. In each simulation the fleet first forms a stable circular formation with center at $\mathbf{c}(0)$ via the inner control loop. It then starts the search mission by implementing the outer control loop. In each simulation $T_{\text {total }}$ is the time duration between the starting search mission at $\mathbf{c}(0)$ and the first time that the center of formation enters into the ball with center $\mathbf{c}^{*}$ and radius $r_{0}=20 \mathrm{~m}$ and stays in this ball for at least $T_{0}=10 T_{a}$ second. Fig. 5 illustrates the trajectory of the center of formation $\mathbf{c}$ subject to 50 percent packet dropout (i.e., $\alpha=0.5$ ) in communication from the fleet to the ASV and 5 percent packet dropout in inter-AUV communication as well as in communication from the ASV to AUVs. Fig. 5 also illustrates the trajectory of the center of formation $\mathbf{c}$ without dropout (i.e., $\alpha=0$ in communication from the fleet to the ASV and no packet dropout in inter-AUV communication as well as in communication from the ASV to AUVs). Both cases do not include any repetition in coding. That is, $M=1$, which corresponds to the two-loop control policy of [3]. As clear from Fig. 5, the trajectory of the case with dropout but without repetition in coding is non-smooth and has a trend to be unstable ${ }^{9}$, while for the case without dropout the source is located in $T_{\text {total }}=4262$ second.

\begin{tabular}{|c|c|c|}
\hline $\mathbf{c}^{*}=\left(\begin{array}{c}154.3 \mathrm{~m} \\
1852.08 m\end{array}\right)$ & $\mathbf{c}(0)=\left(\begin{array}{c}-135 \mathrm{~m} \\
1451.3 \mathrm{~m}\end{array}\right)$ & $\kappa=100$ \\
\hline $\mathrm{K}=0.001$ & $v_{0}=1 \mathrm{~m} / \mathrm{s}$ & $R_{0}=50 \mathrm{~m}$ \\
\hline$V_{\max }=0.15 \mathrm{~m} / \mathrm{s}$ & $T_{s}=0.059 \mathrm{sec}$. & $\mathrm{h}=1500 \mathrm{~m}$ \\
\hline
\end{tabular}

TABLE IV

PARAMETERS USED FOR SIMULATIONS.

As clear from Fig. 5, without repetition in coding, localization of the unknown underwater flow source is not achieved.

As discussed in Section III, a larger $M$ results in a better quality of communication but larger packets transmission delay (and therefore, longer time latency) that both affect the performance of the fleet $T_{\text {total }}$ in the opposite direction: As clear from Fig. 5 for the case of $\alpha=0$, an excellent quality of communication results in a smooth search trajectory for the fleet under

\footnotetext{
${ }^{8}$ To model a typical underwater search mission, the parameters used in simulations, such as the description for concentration flow intensity, $v_{0}, R_{0}$, etc., were chosen following several technical discussions with our colleagues from Ifremer who have many years of practical experience in operational exploration with AUVs.

${ }^{9}$ For this case, after the center of formation enters into the ball with center $\mathbf{c}^{*}$ and radius $r_{0}$, the center of formation shortly exits the ball.
} 


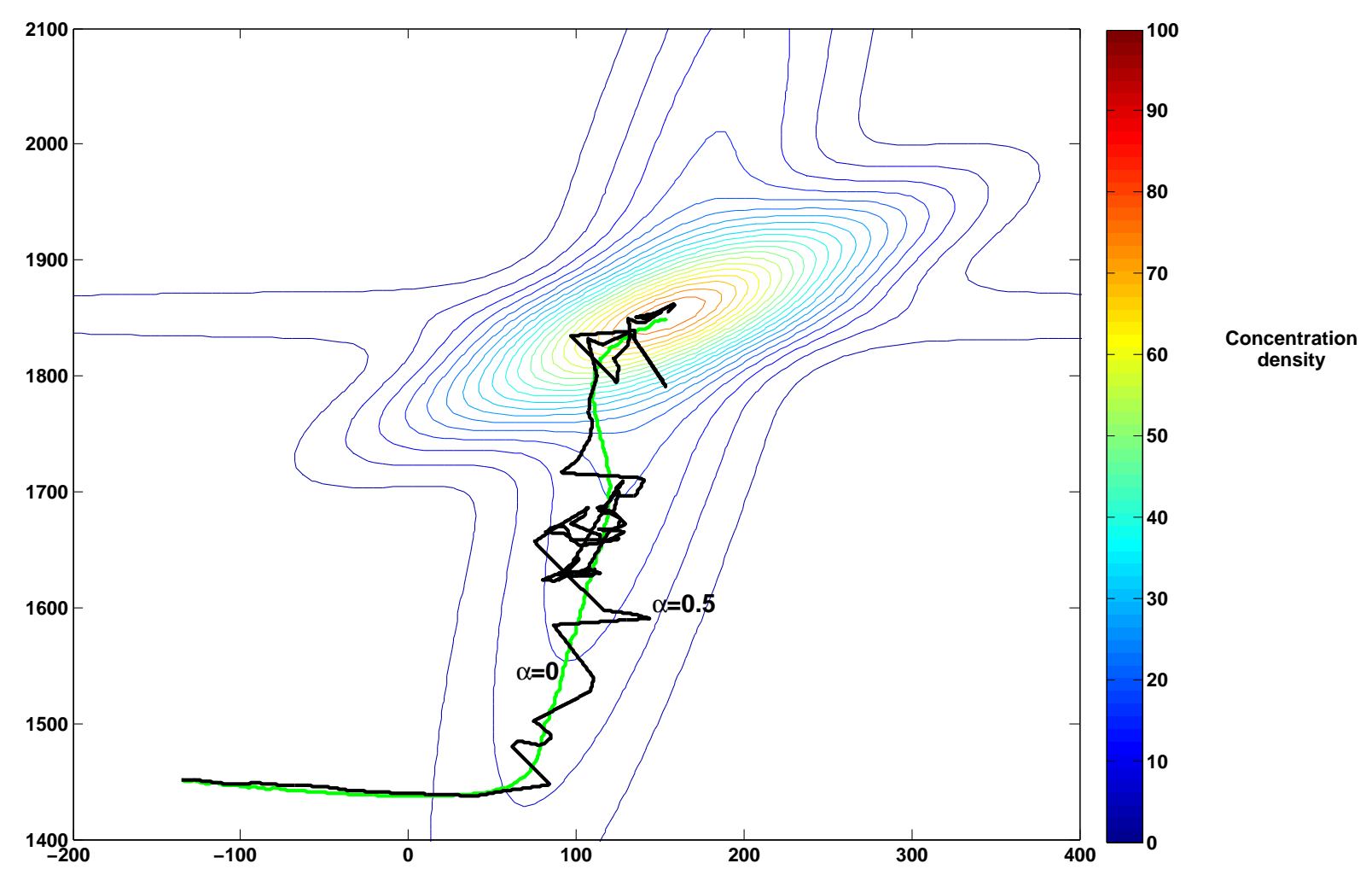

Fig. 5. The trajectory of the center of formation c subject to random packet dropout and no repetition in coding (the black trajectory) and the trajectory of the center of formation $\mathbf{c}$ in the absence of dropout (the green trajectory).

\begin{tabular}{|c|c|c|c|c|c|c|c|}
\hline$M$ & 2 & 3 & 4 & 5 & 6 & 7 & 8 \\
\hline$T_{\text {total }}$ & 8211 & 5798 & 5414 & 6574 & 8123 & 9271 & 10466 \\
\hline
\end{tabular}

TABLE V

THE TRADE-OFF BETWEEN EMPIRICAL $T_{\text {total }}$ MEASURED IN SECOND AND $M$ FOR $\alpha=0.5$. THE PAIR $\left(M^{*}, T_{\text {total }}^{*}\right)=(4,5414)$ IS THE OPTIMUM PAIR.

control. On the other hand, as pointed out in the previous section, a large $M$ results in a large packets transmission delay; and therefore, a long time latency in the outer control loop and a long sampling period $T(M)$. As will be illustrated shortly, this will result in a non-smooth search trajectory and therefore a large $T_{\text {total }}$. Hence, the interactions between quality of communication, transmission delay and control must be balanced via a proper selection of the number of packet repetitions $M$ so that the best performance (which corresponds to the smallest $T_{\text {total }}$ ) is achieved.

To find the proper number of packet repetitions $M^{*}$ for the condition simulated, the simulations are repeated 20 times for each number of packet repetitions: $M=1,2,3,4,5,6,7,8$. For $M=1$, 


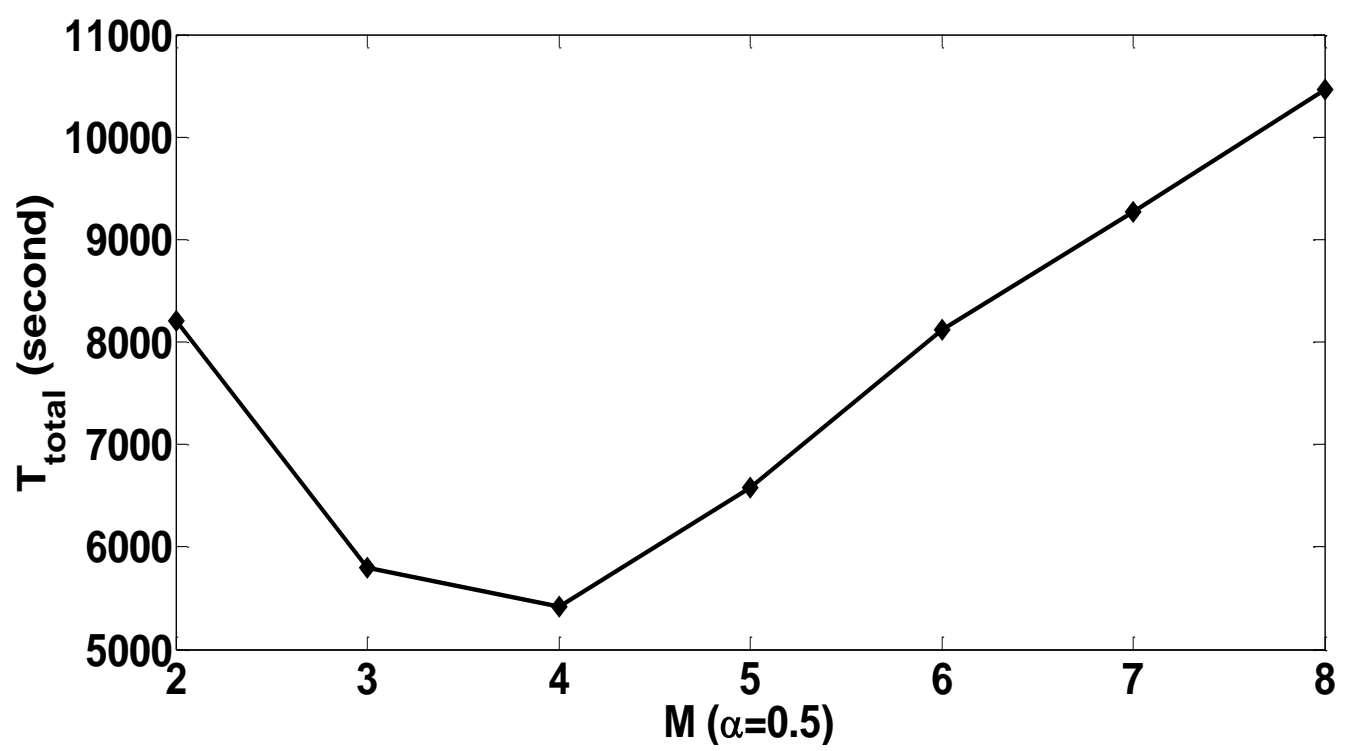

Fig. 6. The trade-off between the mission time $T_{\text {total }}$ and M. From this trade-off it follows that $M^{*}=4$ is the optimum number of packet repetitions.

it is observed that in most cases the source cannot be located and the trajectory of the center of formation has a trend to be unstable ${ }^{10}$. This result is expected from the formula (8) as this formula for $\alpha=0.5$ is not satisfied for $M=1$. For other cases (i.e., $M \geq 2$ ), it is observed that in all simulations, the search is concluded, as expected from the formula (8), within the life time of AUVs which is 5 hour. Table V summarizes the obtained empirical values for the mission time ${ }^{11}$ for different $M$; and Fig. 6 illustrates the trade-off between empirical $T_{\text {total }}$ and $M$. Fig. 7 also illustrates trajectories of the center of formation $\mathbf{c}$ for $M=1,4,8$.

As clear from these figures and the table, the best performance in the presence of random packet dropout and marine perturbation is achieved for $M^{*}=4$. This case corresponds to a communication with good quality (i.e., 100. $\alpha^{M^{*}}=6$ percent packet dropout in communication from AUVs to the ASV) with a acceptable packets transmission delay $\left(C\left(M^{*}\right)=2.4\right.$ second), which results in the best performance, i.e., $T_{\text {total }}^{*}=5414$ second. This performance is only 27 percent worse than the ideal case of no dropout (i.e., $\alpha=0$ and no packet dropout in inter-AUV communication as well as in communication from the ASV to AUVs), which is $T_{\text {total }}=4262$

\footnotetext{
${ }^{10}$ For this case, after the center of formation enters into the ball with center $\mathbf{c}^{*}$ and radius $r_{0}$, the center of formation shortly exits the ball.

${ }^{11}$ For each $M$, the empirical value of mission time (empirical $T_{\text {total }}$ ) is the average of the mission times obtained from simulations.
} 


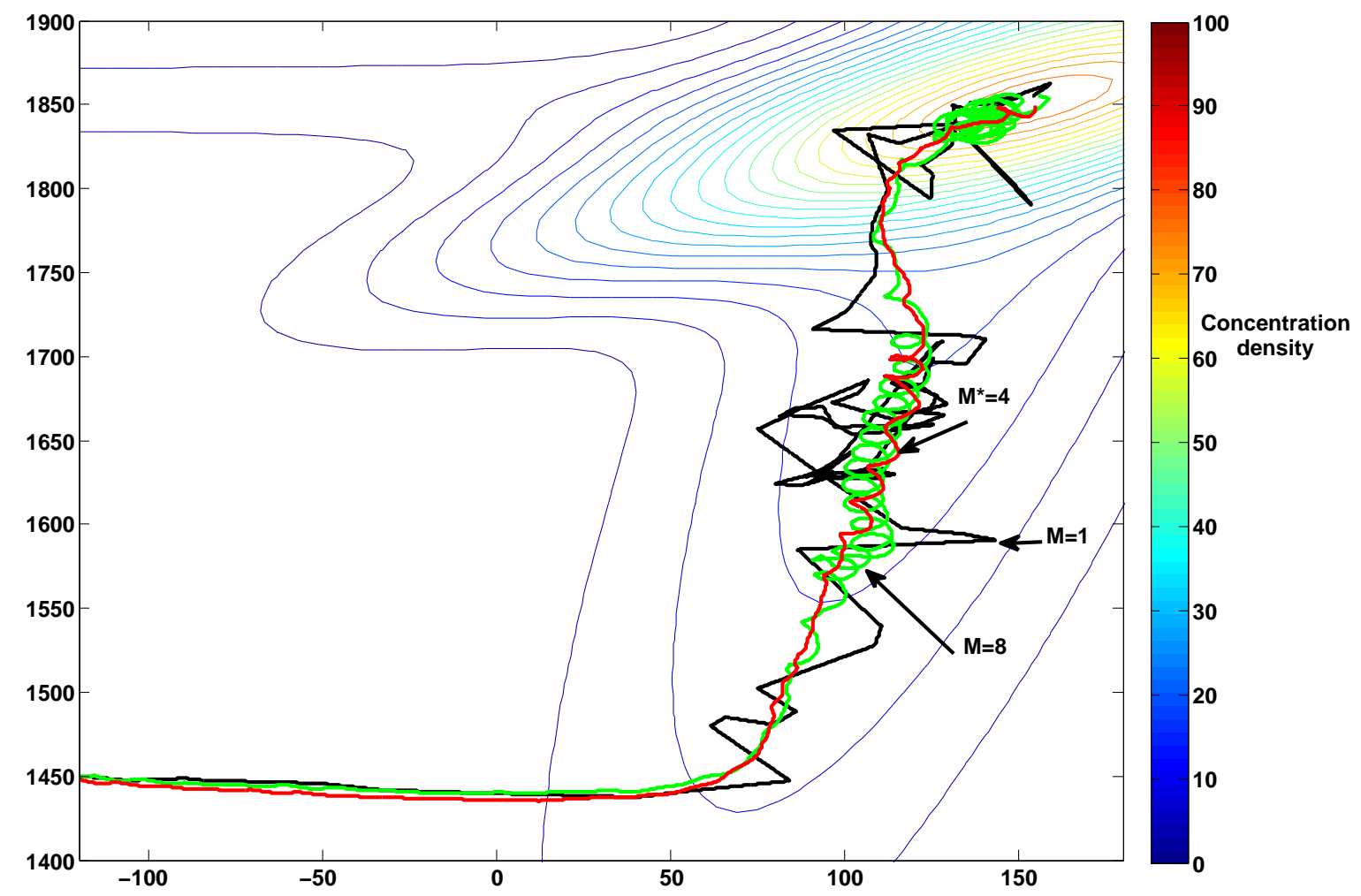

Fig. 7. The trajectories of the center of formation c subject to 50 percent packet dropout in communication from AUVs to the ASV and 5 percent packet dropout in inter-AUV communication as well as in communication from the ASV to AUVs.

second.

The case of $M=1$ corresponds to a system with a poor quality of communication (i.e., 100. $\alpha^{1}=50$ percent packet dropout) but a small packets transmission delay $(C(1)=0.6$ second), in which this poor quality of communication results in a very non-smooth search trajectory. On the other hand, the case of $M=8$ corresponds to a system with an excellent quality of communication (i.e., 100. $\alpha^{8} \approx 0$ percent packet dropout) but large packets transmission delay $(C(8)=4.8$ second), which induces a large time latency of 22.2875 second in the outer control loop. As clear from Fig. 7, this long time latency also results in a non-smooth search trajectory. Therefore, for these two cases the performance is not satisfactory.

For illustration, the simulations are repeated for $\alpha=0.3$ and $\alpha=0.1$. Table VI summarizes the trade-off between empirical mission time and $M$; and Fig. 8 illustrates the trade-off between empirical $T_{\text {total }}$ and $M$. It is observed that for all $M \in\{1,2,3, \ldots, 8\}$ the search is concluded, as expected by the formula (8). From this table and figure, it follows that when $\alpha$ is small (e.g., 


\begin{tabular}{|c|c|c|c|c|c|c|c|c|}
\hline$M$ & 1 & 2 & 3 & 4 & 5 & 6 & 7 & 8 \\
\hline$T_{\text {total }}(\alpha=0.3)$ & 6684 & 4714 & 4538 & 4651 & 5666 & 7341 & 9229 & 10874 \\
\hline$T_{\text {total }}(\alpha=0.1)$ & 4707 & 4646 & 4559 & 4637 & 5580 & 7357 & 9313 & 10659 \\
\hline
\end{tabular}

TABLE VI

The TRAdE-OFF BetweEn EMPIRICAL $T_{\text {total }}$ MEASURED In SECOND AND $M$. For $\alpha=0.3$ The PAIR $\left(M^{*}, T_{\text {total }}^{*}\right)=(3,4538)$ IS THE OPTIMUM PAIR AND FOR $\alpha=0.1$ THE PAIR $\left(M^{*}, T_{\text {total }}^{*}\right)=(3,4559)$ IS THE OPTIMUM PAIR.
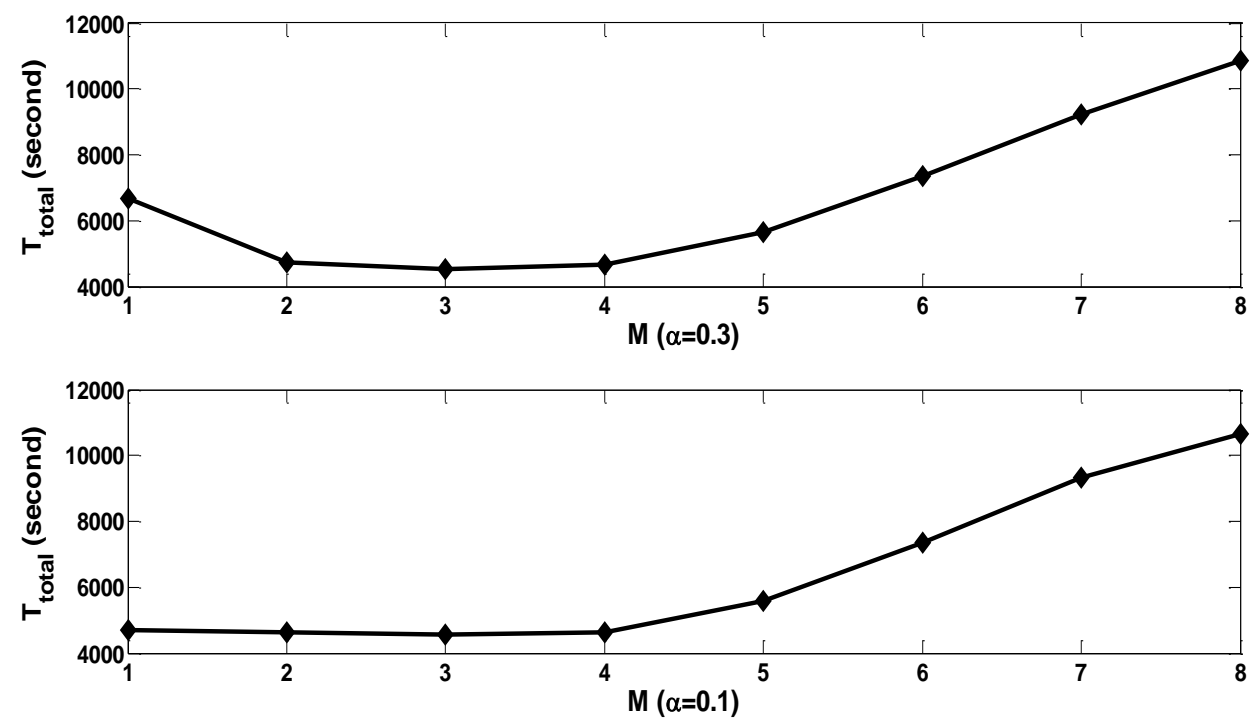

Fig. 8. The trade-offs between the mission time $T_{\text {total }}$ and M. From these trade-offs it follows that $M^{*}=3$ is the optimum number of packet repetitions.

$\alpha \leq 0.1)$ there is no need to use a coding strategy.

\section{CONCLUSiON}

This paper presented an emerging example for the problem of combined control-communication. A supervisory multi - agent control policy over a communication network subject to packet dropout and transmission delay was presented for localization of an underwater flow source with unknown location. It extended the results of [3] to the cases with more realistic representation for concentration flow intensity and illustrated that in the presence of sever random packet dropout the policy that does not consider and compensate the effects of communication imperfection (the policy presented in [3]), cannot stabilize the system (locate the underwater flow source). However, the combined control-communication policy with a good quality of communication and 
relatively low transmission delay, locates the flow source. As illustrated, the proposed controlcommunication policy with a small number of packet repetitions $M$ has a poor quality of communication, which results in a very non - smooth search trajectory. On the other hand, as illustrated, the proposed policy with a very large $M$, which corresponds to a large transmission delay but an excellent quality of communication, does not result in a satisfactory performance either as this case corresponds to a large time latency and a long sampling period for updating the outer control loop action. However, it was illustrated that for $\alpha=0.5, \alpha=0.3$ and $\alpha=0.1$ there is an optimum number of packet repetitions $M^{*}$, which results in the smallest mission time. For future it is interesting to develop theoretical results for finding $M^{*}$ analytically. A starting point to achieve this goal is the development of a theorem that provides an upper bound for $M$ under which the mission time is smaller than the AUVs life time. This theorem combined with Corollary 3.6 determine a set that $M^{*}$ belongs to.

\section{ACKNOWLEDGMENT}

This work is supported by the FeedNetBack European project, grant agreement no.: 223866. The authors thank Lara Brinon Arranz, Alexandre Seuret, Sebastien Varrier, Alain Kibangou, Julien Minet, and colleagues from Ifremer (Jan Opderbecke and Romain Piasco) for many helpful technical discussions.

\section{REFERENCES}

[1] L. Brinon Arranz, A. Seuret, and C. Canudas de Wit, "Translation control of a fleet circular formation of AUVs under finite communication range," in Proc. 48th IEEE Conference on Decision and Control, 2009, pp. 8345-8350.

[2] L. Brinon Arranz, A. Seuret, and C. Canudas de Wit, "Elastic formation control based on affine transformations," in Proc. 2011 American Control Conference, 2011, pp. 3984 - 3989.

[3] B. J. Moore and C. Canudas de Wit, "Source seeking via collaborative measurements by a circular formation of agents," in Proc. 2010 American Control Conference, 2010, pp. 6417-6422.

[4] L. Brinon Arranz, A. Seuret, and C. Canudas de Wit, "Collaborative estimation of gradient direction by a formation of AUVs under communication constraints," in Proceedings of the 50th IEEE Conference on Decision and Control, 2011, pp. 5583-5588.

[5] N. Elia,"When bode meets Shannon: control-oriented feedback communication schemes,", IEEE Trans. Automat. Contr., 49(9), pp. 1477-1488, 2004.

[6] N. Elia and J. N. Eisenbeis,"Limitations of linear control over packet drop networks," IEEE Trans. Automat. Contr., 56(4), pp. 826-841, 2011.

[7] N. C. Martins, A. Dahleh, and N. Elia,"Feedback stabilization of uncertain systems in the presence of a direct link," IEEE Trans. Automat. Contr., 51(3), pp. 438-447, 2006. 
[8] P. Minero, M. Franceschetti, S. Dey, and N. Nair,"Data rate theorem for stabilization over time-varying feedback channels," IEEE Trans. Automat. Contr., 54(2), pp. 243-255, Feb. 2009.

[9] P. Minero, L. Coviello, and M. Franceschetti,“Stabilization over Markov feedback channels: the general case,” IEEE Trans. Automat. Contr., 58(2), pp. 349-362, 2013.

[10] G. N. Nair, R. J. Evans, I. M. Y. Mareels, and W. Moran,“Topological feedback entropy and nonlinear stabilization,” IEEE Trans. Automat. Contr., 49(9), pp. 1585-1597, 2004.

[11] G. N. Nair and R. J. Evans, "Stabilizability of stochastic linear systems with finite feedback data rates," SIAM J. Control Optimization, 43(3), pp. 413-436, 2004.

[12] C. Canudas de Wit, F. Gomez-Estern, and F. Rodrigues Rubio,"Delta-modulation coding redesign for feedback-controlled systems," IEEE Transactions on Industrial Electronics, 56(7), pp. 2684-2696, 2009.

[13] A. Farhadi and N. U. Ahmed,"Tracking nonlinear noisy dynamic systems over noisy communication channels," IEEE Transactions on Communications, 59(4), pp. 955-961, 2011.

[14] S. Varrier, "Robust control of autonomous underwater vehicles," Internship report, in 67 pages, 2010, available at INRIA Rhone - Alpes, Grenoble, France.

[15] A. Goldsmith, Wireless communication. Cambridge University Press, 2005. 